\title{
Avaliação automática de versões oculares em imagens
}

\author{
Jullyana Fialho Pinheiro ${ }^{1}$, João Dallyson Sousa de Almeida ${ }^{1}$,Jorge Antonio \\ Meireles Teixeira $^{1}$, Geraldo Braz Junior ${ }^{1}$
}

${ }^{1}$ Núcleo de Computação Aplicada (NCA)-Universidade Federal do Maranhão,
Av. dos Portugueses, 1966 - Vila Bacanga, São Luís - MA, 65065-545, Brazil
\{jullyanafialho92, jdallyson, jorgemeireles1, ge.braz\}@gmail.com

\begin{abstract}
A vision that comply with the basic visual demands, such as short, long, and lateral vision, needs that ocular muscles work perfectly. People with defective ocular muscles may have several problems including strabismus. This paper presents a method to automate the examination of ocular versions based on images of the patient's face.

Resumo. Uma visão que atenda as demandas visuais básicas como visão a curta e longa distância, visão lateral, entre outras necessita do funcionamento satisfatório dos músculos oculares. Pessoas que possuam algum problema em um dos músculos oculares podem apresentar problemas como o estrabismo. Este trabalho apresenta um método automático para realizar o exame de medida das versões oculares por meio de imagens da face do paciente.
\end{abstract}

\section{Introdução}

Para se obter uma visão que atenda as diversas necessidades visuais (como visão a curta e longa distância, entre outras) é imprescindível o funcionamento de forma eficiente dos músculos oculares [Bicas 2003].

O exame de versão permite a análise desses músculos através da avaliação das posições diagnósticas, estas posições tem como objetivo avaliar o funcionamento dos músculos oculares, são eles: reto superior, reto medial, reto inferior, reto lateral, oblíquo superior e oblíquo inferior.

Geralmente o exame é realizado pelos especialistas de forma subjetiva. Um diagnóstico automatizado visa prover ao oftalmologista um suporte a mais no diagnóstico dos desvios que podem ser apresentados pelos músculos oculares. Assim, este artigo tem como objetivo apresentar um método baseado em imagens para automatizar o exame de versões oculares.

\section{Materiais e Métodos}

O método proposto é formado por 5 etapas: (1) aquisição de imagens e préprocessamento, (2) detecção de olho, (3) localização da pupila, (4) obtenção do formato do olho e (5) medida das versões. A Figura 1 mostra o fluxograma que representa o método.

\subsection{Aquisição e Pré-processamento}

O método será avaliado em uma base formada por imagens de pacientes obtidas no consultório médico, com o paciente sentado, por meio de um smartphone equipado com 
Figura 1. Metodologia proposta.

câmera digital de 16 Megapixels com a função flash desligada e um zoom de 3x e resolução de 1280 x 960. Apenas a área do rosto referente aos olhos é fotografada. Para tratar problemas referentes a variação de iluminação, a imagem adquirida é pré processada utilizando o algoritmo Color Badger [Banić and Lončarić 2014].

\subsection{Detecção de olhos}

Para encontrar os olhos utiliza-se o método apresentado na Figura 2. A região da pele é segmentada (Figura 2c) com o uso do Mapa de Distância de Cor (CDM) [Abdullah-Al-Wadud and Chae 2008], que separa os pixels em pele e não-pele. O maior segmento de pele é obtido gerando uma máscara (Figura 2d). A Figura 2c é subtraída da Figura 2d gerando Figura 2e. Os conjuntos remanescentes são os candidatos a olho. Esses conjuntos tem suas características extraídas pelo HOG (Histograma de gradientes orientados) [Banerji et al. 2013] e em seguida, são classificados utilizando o classificador Random Forest [Breiman 2001].
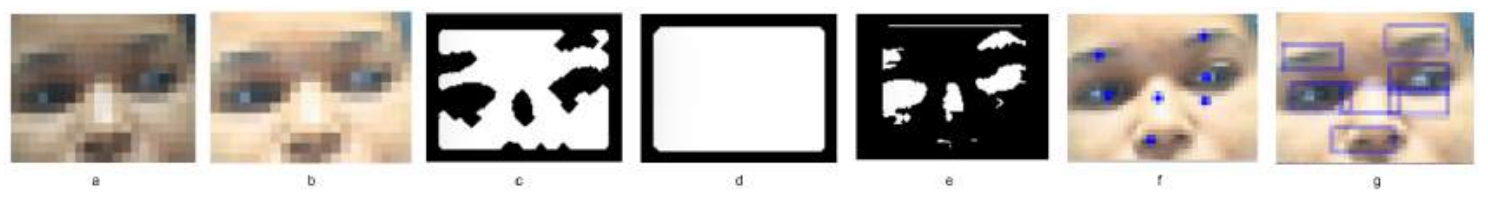

Figura 2. Detecção dos olhos.

A base de imagens de olhos e não olhos (outras regiões da face) utilizadas pelo classificador no treinamento foi obtida em [De Almeida et al. 2015]. Com os olhos localizados as próximas etapas são executas nas regiões referentes aos olhos (Figura 2g).

\subsection{Localização da pupila}

A localização da pupila é realizada em imagens com o modelo de cor HSV. O modelo foi utilizado pois em seus canais $\mathrm{S}$, a região da pupila apresenta valores mais altos quando comparado com seus vizinhos [Zheng et al. 2005]. Logo, se torna possível realizar a localização do ponto central da pupila ao se encontrar esses valores.

Esse ponto é encontrado na realização da binarização da imagem considerando 255 se $\mathrm{f}(\mathrm{x}, \mathrm{y})$ maior ou igual a 240 e 0 caso contrário. Na imagem binarizada aplica-se uma projeção integral vertical e horizontal, os picos de ambas as projeções são utilizados para a formação das coordenadas da pupila. Na ausência de flash na obtenção das imagens, o canal $\mathrm{H}$ pode ser utilizado.

\subsection{Obtenção do Formato do Olho}

A obtenção do formato é executada para que os limites de movimentação dos olhos para a realização da versão sejam obtidos. O método utiliza o canal H do modelo HSV que apresenta baixa intensidade nos pixels referentes aos olhos. 
Nesta imagem é aplicada uma binarização utilizando o limiar de 50 pixels. Com o elemento resultante, o seguinte conjunto de pontos são encontrados: pontos laterais mais distantes, ponto médio entre pontos laterais, os pontos com maior diferença de intensidade na vertical superior e inferior referentes ao centro da pupila. Exemplos desses pontos são mostrados na Figura 3a. Com esses pontos é possível obter o formato dos olhos através da função spline. A Figura $3 \mathrm{~b}$ mostra um exemplo da aplicação do método.

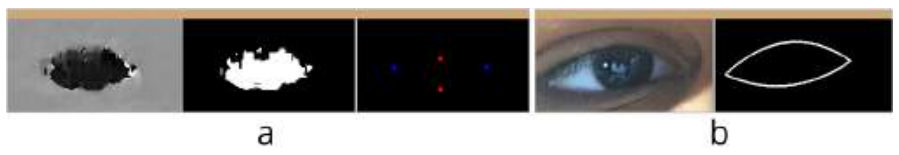

Figura 3. (a) Pontos obtidos com base no formato da imagem binarizada. (b) Resultado do uso da função Spline para formação do contorno dos olhos.

\subsection{Medida das Versões}

As versões são realizadas para a verificação do funcionamento de seis músculos oculares, este artigo apresenta a avaliação da movimentação de dois destes músculos: reto lateral e medial. Para realização desde teste utilizam-se três imagens, uma com o olhar frontal, uma com o olhar direcionado para a direita e outra para a esquerda, Figura 4.
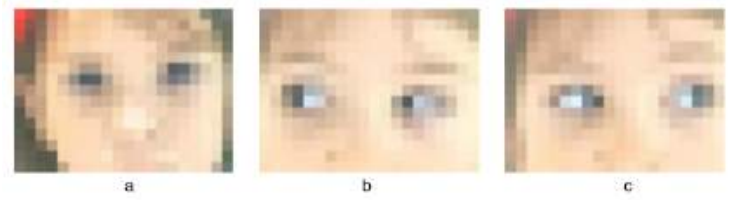

Figura 4. Imagens de exame de versão dos músculos reto lateral e medial.

A medida da versão é obtida através das checagens das seguintes medidas entre os dois olhos: raio da íris, a localização da íris em relação ao ponto médio do olho e discrepância nas distâncias entre o canto do olho verificado (direito ou esquerdo) e a iris. Elas são mostradas na Figura 5 nas cores azul, verde e vermelho respectivamente. A continuação desde trabalho visa classificar a intensidade dos desvios para que o diagnóstico possa auxiliar o planejamento cirúrgico realizado pelo médico.

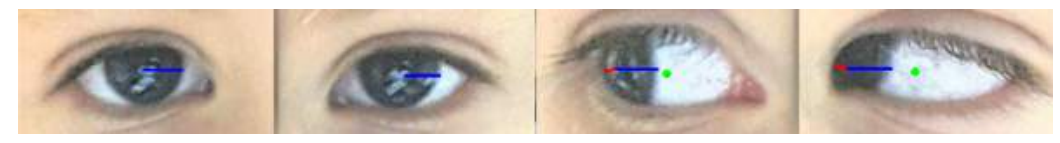

Figura 5. Métricas de desvio nos músculos: reto lateral e reto medial.

\section{Resultados}

Alguns experimentos foram realizados a fim de avaliar a qualidade dos métodos apresentados. A base de dados utilizada é composta por 35 imagens da face de 5 indivíduos, sendo 7 imagens de cada paciente. Das quais, seis correspondem ao testes do exame e uma delas é uma imagem frontal do paciente.

A localização dos olhos foi avaliada em duas partes: (1) a localização de todos os candidatos a olhos para serem classificados e (2) o resultado da classificação. 
Todos os olhos foram localizados e tiveram suas características extraídas. Para validar a classificação dos candidatos a olhos utilizou-se a base de imagens de [De Almeida et al. 2015], por possuir um volume maior de imagens de face (225 imagens), obtendo acurácia de 98,08\%. Já na base de pacientes de versão (Seção 2.1) obtivemos acurácia de $95,95 \%$ com $96 \%$ de precisão e $96 \%$ de especificidade utilizando o modelo treinado com imagens utilizadas na validação.

A obtenção do formato do olho e da localização da pupila tiveram sua eficácia avaliada com base em imagens segmentadas da esclera e da pupila dos olhos respectivamente. Resultados que obtiveram uma área semelhante de segmentação maior que $90 \%$ foram consideradas corretas. A acurácia para essa etapa foi de 94,35\%.

As versões tiveram sua eficacia avaliada pela similaridade entre o resultado do especialista e a encontrada pelo método. Das imagens (10) analisadas apenas um olho apresentou erro, com a discrepância de 1 ponto para o médico. As demais obtiveram o mesmo resultado do médico. A acurácia foi de $95 \%$, medida em 20 olhos analisados.

\section{Conclusão}

Os resultados obtidos apresentam uma perspectivas favorável do método, porém a necessidade de se obter medidas de alta precisão para a correta realização do exame da versão impõe a extensão da base apresentada neste artigo. Com um aumento não só do número de imagens, mas também nos exemplos onde o paciente possuem desalinhamento ocular que possa ser quantificado.

As próximas etapas a serem realizadas serão o aumento do número de imagens de exame de versão, a inserção na base de pacientes com desvios nos músculos reto lateral e reto medial e a realização da verificação e medida de desvios para o restante dos músculos. Os autores agradecem a FAPEMA/CAPES e CNPq pelo apoio.

\section{Referências}

Abdullah-Al-Wadud, M. and Chae, O. (2008). Skin segmentation using color distance map and water-flow property. In 2008 The Fourth International Conference on Information Assurance and Security, pages 83-88.

Banerji, S., Sinha, A., and Liu, C. (2013). Haarhog: Improving the hog descriptor for image classification. In 2013 IEEE International Conference on Systems, Man, and Cybernetics, pages 4276-4281.

Banić, N. and Lončarić, S. (2014). Color Badger: A Novel Retinex-Based Local Tone Mapping Operator, pages 400-408. Springer International Publishing, Cham.

Bicas, H. E. A. (2003). Oculomotricidade e seus fundamentos. Arquivos Brasileiros de Oftalmologia, 66:687 - 700.

Breiman, L. (2001). Random forests. Machine Learning, 45(1):5-32.

De Almeida, J. D. S., Silva, A. C., Teixeira, J. A. M., Paiva, A. C., and Gattass, M. (2015). Computer-aided methodology for syndromic strabismus diagnosis. Journal of digital imaging, 28(4):462-473.

Zheng, Z., Yang, J., and Yang, L. (2005). A robust method for eye features extraction on color image. Pattern Recognition Letters, 26(14):2252 - 2261. 